Boll. Accademia Gioenia di

Scienze Naturali - Catania
BOLLAG Vol. 52, N. 382 (2019)

Difesa ecosostenibile delle colture agricole pp. DECA10 - DECA16

ISSN 0393-7143

Anno di fondazione 1824

\title{
Il miglioramento genetico per la resistenza agli insetti: una sfida difficile ed attuale ${ }^{\dagger}$
}

\author{
A. Gentile \& S. La Malfa [1]* \\ Dipartimento di Agricoltura, Alimentazione e Ambiente, Università degli studi di Catania
}

\begin{abstract}
Riassunto
Nello scenario di una nuova agricoltura, le tecniche di miglioramento genetico sono chiamate a svolgere un ruolo importante al fine di rendere i processi produttivi sostenibili anche sotto il profilo ambientale ed economico. Nei prossimi anni sarà necessario produrre di più in condizioni di risorse naturali decrescenti e al tempo stesso assicurare produzioni anche attraverso una riduzione degli input chimici. Negli ultimi cinquant'anni l'integrazione di diverse tecniche e lo sviluppo di metodi di rigenerazione in vitro e di strategie molecolari ha consentito la definizione di nuovi strumenti di miglioramento genetico. Le conoscenze genomiche degli ultimi decenni hanno, infatti, innovato gli strumenti per la realizzazione di programmi di miglioramento genetico, sia in termini di disponibilità di marcatori per selezione assistita o per selezione genomica, sia in termini di conoscenze sulla funzione dei geni responsabili di caratteri agronomici fondamentali, tra i quali la resistenza a stress biotici. I nuovi metodi molecolari, che consentono di ottenere piante cisgeniche, o con sequenze modificate mediante genome editing, si affiancano ai metodi tradizionali di miglioramento genetico superando i limiti dell'incrocio e della selezione, soprattutto con riferimento alla lunghezza dei tempi ed alla impossibilità di prevederne il risultato in termini di caratteristiche modificate. Nella nota vengono descritti i principali metodi di miglioramento genetico e le principali strategie utilizzabili per migliorare la resistenza delle piante agli insetti.
\end{abstract}

Parole chiave: DNA, transgenesis, new breeding techniques, resistance, pests

\section{Breeding for pest resistance: a difficult and current challenge}

\section{Summary}

In order to face the new challenges of agriculture, genetic improvement is called to play an important role for making production processes sustainable also from an environmental and economic point of view. In the coming years it will be necessary to produce more but in a scenario of decreasing natural resources and also with a reduction of chemical inputs. In the last fifty years, the integration of different techniques and the development of in vitro regeneration methods and molecular strategies has allowed the definition of new tools for genetic improvement. The genomic knowledge of the last decades have, in fact, innovated the tools for the development of programs of genetic improvement, both in terms of availability of markers for assisted selection or for genomic selection, and in terms of knowledge on the function of genes responsible for specific agronomic traits such as the resistance to biotic stress. These molecular methods allow to obtain cisgenic plants, can help to modify target sequences through genome editing, and can be considered as

\footnotetext{
${ }^{\dagger}$ Nota presentata nell'Adunanza pubblica del 15 novembre 2018

*e-mail: stefano.lamalfa@unict.it
} 
complementary to traditional breeding methods. At the same time, they can overcome some of the limits of crossing and selection, especially those related to their long duration and the unpredictability of the results in terms of changed characteristics. In this contribution we describe the main methods of genetic improvement and the main strategies that can be used to improve the resistance of plants to insects.

Key words: DNA, transgenesis, new breeding techniques, resistenza, parassiti

\section{Introduzione}

La disponibilità di varietà migliorate per caratteri di interesse agronomico, rappresenta tuttora uno dei fattori chiave per lo sviluppo e l'affermazione dell'agricoltura a livello mondiale [1]. Attraverso il miglioramento genetico, sia per le specie a propagazione vegetativa, sia per quelle a propagazione gamica, ci si prefigge di adattare quanto più possibile la pianta all'ambiente che la ospita, rendendo possibile il successo agronomico della coltivazione. Una scelta consapevole della varietà da coltivare permette di soddisfare la vocazionalità ambientale consentendo di riscontrare le esigenze delle colture e di adattarle all'ambiente ove vengono coltivate, anche con riferimento ai nuovi scenari determinati dai cambiamenti e dalla variabilità climatica; quest'ultimo aspetto è peraltro strettamente connesso a quella della diffusione di nuovi patogeni e parassiti. Da questa breve premessa emerge chiaro il ruolo chiave che il miglioramento genetico assume nella moderna agricoltura, ed è agli sviluppi di questo settore che spesso si fa riferimento parlando di "nuova rivoluzione verde", anche in relazione alle innovative tecniche utilizzabili, note con il termine di New Breeding Techniques, NBTs [2].

In questo contributo saranno descritte le principali tecniche di miglioramento genetico delle specie di interesse agrario con particolare riferimento alla resistenza a stress biotici e a come le conoscenze derivanti dal settore della genomica possano consentire di abbreviare i tempi necessari per il raggiungimento di specifici obiettivi.

\section{Metodi di miglioramento genetico}

Schematicamente i metodi di miglioramento possono essere suddivisi in metodi classici e metodi innovativi; questi ultimi vengono così definiti poiché nel corso della loro realizzazione si avvalgono dell'utilizzo di tecniche di laboratorio. Questa suddivisione potrebbe tuttavia trarre in inganno il lettore ove non si considerasse che in realtà molte tecniche avanzate (coltura in vitro, analisi del DNA), fanno oggi parte in maniera consolidata del patrimonio di conoscenze di chi si occupa di miglioramento genetico [3]. Tra i metodi classici, vanno considerati l'incrocio, la selezione clonale e la mutagenesi.

L'incrocio, in tutte le sue variabili (autofecondazione, ibridazione intra- ed inter-specifica, od anche intergenerica, reincrocio), rappresenta senza dubbio il metodo di miglioramento genetico più utilizzato. La scelta dei parentali è una fase cruciale per il buon esito di un programma di ibridazione e particolare attenzione deve essere posta alla conoscenza dei caratteri specifici che si intendono migliorare nella progenie, soprattutto per i caratteri quantitavi (poligenici). L'incrocio, pur essendo effettuato mediante l'applicazione di schemi classici, si avvantaggia delle informazioni oggi disponibili soprattutto con riferimento alla conoscenza della base genetica dei caratteri ed alla possibilità di applicare la cosiddetta selezione assistita da marcatori (Marker Assisted Selection - MAS) per lo screening delle popolazioni ibride ottenute.

La selezione clonale ha invece l'obiettivo di individuare e selezionare i migliori cloni presenti all'interno delle varietà coltivate. Le principali caratteristiche migliorative che è possibile fissare riguardano produttività, qualità complessiva del frutto, epoca di maturazione. Minori sono invece

le possibilità di intervento sulle caratteristiche di resistenza ad avversità biotiche. Nel processo di 
selezione clonale, un ruolo decisivo viene svolto dagli operatori di campo per la individuazione, all'interno di impianti omogenei, di piante con caratteristiche superiori.

La mutagenesi si basa sulla possibilità di fissare, attraverso la propagazione vegetativa, una quota di variabilità che può insorgere, naturalmente o artificialmente, nelle varietà coltivate. L'insorgenza di variabilità può essere incrementata attraverso il ricorso a tecniche induttive; queste consistono nell'applicazione di agenti mutageni fisici o chimici quali la colchicina o i raggi gamma, i raggi X e gli isotopi del Cobalto, utilizzati su porzioni di piante che vengono successivamente propagate. Un aspetto particolare della mutagenesi riguarda la stabilità delle mutazioni da selezionare. Non sono infatti rari i casi di regressione delle mutazioni e di conseguente ricomparsa del carattere originario.

Più di recente, i progressi della biologia molecolare hanno dato un decisivo impulso alla ricerca in agricoltura, rendendo possibile la individuazione e la manipolazione di geni di interesse agronomico. Parimenti anche le tecniche di manipolazione tramite coltura in vitro di organi diversi (tessuti, protoplasti, antere etc.) hanno reso possibile lo sviluppo di interventi di miglioramento genetico quali l'ibridazione somatica, la selezione in vitro, la trasformazione genetica (Fig. 1); oggi, le tecniche di miglioramento genetico traggono vantaggio anche da tecnologie di sequenziamento di nuova generazione (NGS) che hanno reso possibile il sequenziamento di svariate decine di genomi di specie di interesse agrario.

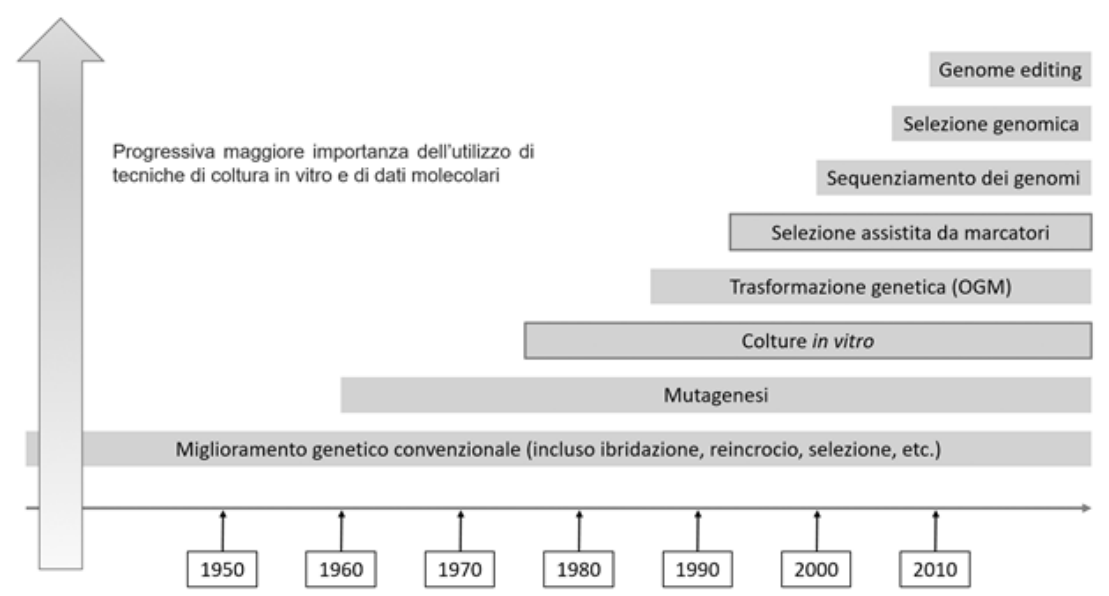

Fig. 11 - Evoluzione dei metodi e delle biotecnologie per il miglioramento genetico vegetale. Modificata da Cattivelli e Valè, 2017 [2]

Tra le tecniche più efficaci di miglioramento genetico, la trasformazione genetica è certamente tra le più significative consentendo di trasferire ed integrare stabilmente all'interno del genoma di un organismo specifici geni. I prodotti di questo metodo di miglioramento genetico prendono il nome di OGM (organismi geneticamente modificati) o PGM (piante geneticamente modificate). A livello di definizione, in accordo con la direttiva europea 2001/18, un OGM è "un organismo il cui materiale genetico è stato modificato in modo diverso da quanto avviene in natura con l'accoppiamento e/o ricombinazione genetica naturale". Le prime piante geneticamente modificate o transgeniche furono ottenute agli inizi degli anni ' 80 e da allora sono state molteplici le applicazioni in termini di tratti e di specie e varietà modificate. Le potenzialità della tecnica sono tali da consentire applicazioni che travalicano il perimetro di interesse legato all'impiego alimentare delle piante coltivate. Le piante geneticamente modificate, e tra queste soprattutto soia, cotone e mais, sono state oggetto per oltre 20 anni di crescente diffusione in alcune zone del pianeta che non includono il nostro Paese e buona parte dei Paesi dell'Unione Europea. Varietà geneticamente modificate possono essere ottenute mediante due diversi metodi: uno indiretto basato sull'utilizzo dell'agrobatterio (Agrobacterium tumefaciens) che funge da vettore del DNA di interesse all'interno delle cellule, ed uno diretto che prevede l'inserimento del DNA attraverso il metodo biolistico. Per entrambi i metodi, presupposto fondamentale è la disponibilità di efficienti protocolli di coltura 
in vitro. Il metodo di trasferimento del DNA basato su Agrobacterium tumefaciens (agente responsabile in natura di una malattia nota come "tumore del colletto") sfrutta la capacità del batterio di trasferire porzioni del suo patrimonio genetico, contenuti all'interno dei plasmidi (molecole circolari di DNA), ad altri organismi viventi. I moderni plasmidi si dicono "disarmati”, perché privi dei geni patogeni, ed "ingegnerizzati", perché contengono il gene di interesse con annesse le sequenze regolatrici indispensabili per il suo funzionamento all'interno delle cellule ospiti; contengono inoltre geni marcatori, selezionabili o reporter, utili per consentire il processo di selezione delle cellule trasformate o per monitorarlo. Il processo di trasformazione, includendo fasi di coltura in vitro ed interazione tra due organismi viventi, è soggetto all'influenza di numerosi fattori che lo rendono particolarmente complesso e genotipo-dipendente.

Nell'Unione Europea il quadro normativo che regola il rilascio di OGM a fini di sperimentazione e di commercializzazione è piuttosto articolato, essendosi nel tempo stratificate diverse norme. Un elemento di chiarezza proveniva dalla Raccomandazione della Commissione Europea del 23 luglio 2003 che sanciva che "Nell'UE non deve essere esclusa alcuna forma di agricoltura, convenzionale, biologica o che si avvale di OGM" ed a tali principi sembrava essere ispirata la legge 5/2005 del nostro Paese che però, demandando alle singole Regioni l'adozione dei piani di coesistenza e l'identificazione di siti pubblici dove poter attivare la sperimentazione, ha nei fatti prolungato i tempi della moratoria sulla sperimentazione vigente dal 2001. Le remore rispetto all'utilizzo degli OGM in agricoltura sono numerose e includono obiezioni di principio e di merito. Le prime riguardano una generale critica alle biotecnologie ed a tutti gli interventi dell'uomo sulla natura, spesso basata su una scarsa conoscenza dei fenomeni naturali legati alla evoluzione, alla storia e all'impatto del miglioramento genetico, anche di quello più "tradizionale". Le obiezioni di merito sollevano invece importanti questioni e meritano una attenta valutazione, anche per l'impulso che possono dare verso nuove soluzioni ed applicazioni.

Alla luce delle attuali conoscenze occorre riflettere sul ruolo che lo sviluppo delle biotecnologie vegetali (soprattutto le New Breeding Techniques che saranno brevemente descritte in seguito) possono avere per risolvere problemi specifici della nostra agricoltura. Di fatto molto spesso il breeder si trova di fronte alla necessità di modificare un singolo tratto (ad esempio una resistenza) in una varietà selezionata senza alterare il complesso funzionale di geni che rendono quella determinata varietà di pregio. Il miglioramento genetico si è da sempre avvalso di modificazioni ottenute con metodi diversi. Dalla selezione di mutazioni casuali, si è passati all'uso dell'incrocio ed alla mutagenesi indotta. In una seconda fase i breeder hanno potuto indurre variabilità mediante tecniche diverse sino all'ottenimento delle piante geneticamente modificate. Negli ultimi anni, l'ulteriore progresso delle conoscenze, soprattutto a livello genomico, ha consentito di effettuare interventi sempre più mirati perché basati sulla conoscenza a livello di sequenza nucleotidica dei geni di interesse fino alla messa a punto delle New Breeding Techniques (NBTs). Tra queste, la cisgenesi è una tecnica di trasformazione genetica che consente di ottenere un prodotto identico a quello ottenibile attraverso l'incrocio "naturale" poiché prevede il trasferimento di geni provenienti da una specie o varietà interfertile con quella ricevente. Si noti che il sistema di inserimento del gene (anche in questo caso diretto o indiretto) non prevede l'inserzione di ulteriori sequenze regolatrici o marcatori. Allo stato attuale delle conoscenze la cisgenesi appare utile soprattutto per il trasferimento di geni di resistenza a patogeni e difatti, tra i primi risultati applicativi di un certo interesse, va ricordato l'ottenimento di varietà di melo resistenti a ticchiolatura (la più grave malattia crittogamica del melo, la cui gestione richiede numerosi interventi di lotta chimica), utilizzando il gene di resistenza presente nella specie Malus floribunda, specie selvatica sessualmente compatibile con le varietà coltivate appartenenti alla specie $M$. domestica.

La seconda tecnica NBT fa riferimento al "genome editing", letteralmente "modifica del linguaggio di sequenza" attraverso il quale è possibile modificare la sequenza dei nucleotidi determinando mutazioni del tutto simili a quelle ottenibili attraverso il processo di mutagenesi, ma in maniera mirata. Presupposto di tale tipo di intervento è la precisa conoscenza della sequenza del gene o della sua forma allelica responsabile di un determinato carattere e degli effetti che la sua modifica può determinare sul fenotipo della varietà considerata. 
La modificazione può essere indotta attraverso l'utilizzo di enzimi (nucleasi) che generano rotture nel doppio filamento, ed in particolare attraverso il sistema denominato CRISPR associato all'enzima CAS9 (CRISPR/CAS9) che permette di ottenere elevate frequenze di mutazione e che potrebbe consentire di introdurre, in varietà coltivate (spesso dotate di un background genetico molto valido), una qualsiasi mutazione favorevole osservata in altri genotipi, senza che questo comporti l'inserimento di nuovi geni e il riassortimento complessivo del genoma che a sua volta renderebbe indispensabile il ricorso a lunghi cicli di reincrocio per reintegrare le caratteristiche di pregio presenti nelle varietà coltivate.

Per sfruttare appieno le potenzialità del genome editing saranno necessari ovviamente progressi nell'identificazione dei geni da mutagenizzare per modificare i caratteri di interesse, e nella capacità di rigenerare in vitro in maniera quanto più indipendente dal genotipo, come già discusso a proposito delle tecniche di trasformazione genetica. Rispetto alla tecnologia transgenica, entrambe le tecniche descritte presentano il vantaggio di non prevedere l'introduzione di alcun tipo di sequenza estranea nel genoma della varietà da migliorare.

\section{Obiettivi del miglioramento genetico e specificità di quello per la resistenza a insetti}

Per ciascuna specie coltivata sono molteplici gli scopi che il miglioramento genetico può perseguire con l'obiettivo di risolvere problemi dettati da condizioni esterne alla coltura (stress di varia natura), di adattare la stessa a mutate condizioni, di migliorarne l'efficienza produttiva, di diversificarne l'epoca di maturazione, di migliorare specifiche caratteristiche qualitative del frutto o la sua funzionalità d'uso (Tabella 1).

\begin{tabular}{|l|}
\hline Miglioramento dell'adattabilità all'ambiente \\
\hline Aumento della produttività \\
\hline Estensione degli areali di coltivazione \\
\hline Introduzione di resistenze a stress biotici ed abiotici \\
\hline Miglioramento di tratti qualitativi della produzione \\
\hline Estensione del calendario di maturazione \\
\hline Aumento della shelf-life \\
\hline Diversificazione della tipologia di prodotto \\
\hline Aumento delle proprietà salutistiche \\
\hline Modificazione di habitus vegetativo \\
\hline Modifica di particolari tratti coinvolti nella biologia riproduttiva \\
\hline
\end{tabular}

Tabella 1 - Principali obiettivi del miglioramento genetico delle piante coltivate

Dal punto di vista del breeder, la resistenza agli insetti rappresenta un obiettivo che può essere perseguito attraverso diverse strategie e diversi metodi, ma vi sono da tenere in considerazione alcune specificità poiché nel meccanismo di resistenza risultano coinvolti due differenti sistemi genetici (dell'ospite e dell'agente responsabile della malattia), nonché la loro interazione. L'intervento di breeding presuppone pertanto la conoscenza dei rapporti che si instaurano tra i due esseri viventi e come questi si sono coevoluti nel tempo e nello spazio. Infatti la reale resistenza ha una base genetica e solo in quanto tale è di interesse ai fini del breeding, sia che ci si trovi di fronte ad un carattere di tipo quantitativo (monogenico) o, più frequentemente, di tipo qualitativo (poligenico). Il miglioramento genetico per la resistenza ad insetti inoltre può rappresentare tanto un obiettivo diretto, qualora sia finalizzato a limitare il danno alle colture, quanto un obiettivo indiretto, quando è finalizzato al controllo di insetti vettori di altre malattie quali quelle particolarmente temibili indotte da virus o da batteri (Virus della tristeza e Huanglongbing negli agrumi, Xylella nell'olivo, giusto per citare alcuni esempi tra i più noti) [4]. 
Rispetto a tutte le altre strategie di lotta e di controllo degli insetti, l'utilizzo di varietà resistenti è vantaggioso per molteplici motivi. Tale approccio infatti consente un controllo a lungo termine della problematica, ha un minor costo per l'agricoltore rispetto agli interventi di campo, è compatibile con le altre forme di controllo, ivi inclusi gli approcci di controllo biologico, non ha ripercussioni dirette sull'ambiente e risulta sicuro per l'uomo. Ovviamente non mancano risvolti di segno negativo rappresentati principalmente dai tempi, generalmente molto lunghi, necessari per l'individuazione delle fonti di resistenza e per il loro trasferimento, e dalla ricorrenza di fenomeni di evoluzione dei fitofagi e conseguente superamento dei meccanismi di difesa delle piante; tali meccanismi di adattamento sono frequenti soprattutto nel caso in cui la resistenza sia determinata da un carattere monogenico. Nel caso in cui un nuovo biotipo di un fitofago riesca a superare i meccanismi di resistenza, i programmi di miglioramento genetico devono riorientarsi alla ricerca di nuovi geni di resistenza.

Esistono in letteratura alcuni esempi particolarmente significativi di successo nella costituzione di genotipi resistenti ad attacchi di insetti fitofagi. In frumento sono ad esempio state costituite già a partire dagli anni ' 50 del secolo scorso varietà resistenti alla Cecidomia distruttrice del frumento (Mayeticola destructor). Tali varietà sono state ottenute attraverso l'incrocio delle varietà coltivate con alcune linee selvatiche resistenti. Al tempo stesso la storia della resistenza a questo dittero riporta anche del frequente superamento dei meccanismi di resistenza nella varietà ottenuta per cui il lavoro di miglioramento genetico prosegue utilizzando fonti di resistenza fortunatamente presenti in specie e generi affini al Triticum, oltre che prevedendo strategie idonee a limitare $\mathrm{i}$ meccanismi di adattamento dell'insetto [5,6]. Ma l'esempio maggiormente conosciuto riguarda probabilmente la Fillossera della vite (Phylloxera vastatrix). L'attacco di questo afide è esiziale per le radici della vita europea, mentre i suoi effetti dannosi sono attenuati sulle specie di vite autoctone del continente americano (V. riparia, V. rupestris, $V$. berlandieri, soprattutto) alle quali si fece ricorso come fonti di resistenza in quanto tali, o attraverso loro selezioni o incroci, dopo l'introduzione della fillossera in Europa sul finire del 1800 [7]. Ulteriore esempio di trasferimento di caratteri di resistenza, in questo caso a nematodi, è rappresentato da alcuni portinnesti di drupacee quali il Nemaguard (Prunus persica $x$ Prunus davidiana) [8].

\begin{tabular}{|c|c|c|}
\hline Coltura e specie & Geni & Parassita \\
\hline \multirow{2}{*}{$\begin{array}{l}\text { Frumento } \\
\text { (Triticum aestivum) }\end{array}$} & $\begin{array}{l}\text { Diversi geni } H \\
\text { (risposta di ipersensitività) }\end{array}$ & $\begin{array}{l}\text { Cecidomia distruttrice del frumento } \\
\text { (Mayetiola destructor) }\end{array}$ \\
\hline & $\begin{array}{l}\text { Diversi geni } D n \\
\text { (resistenza ad afidi) }\end{array}$ & $\begin{array}{l}\text { Afide russo del frumento } \\
\text { (Diuraphis noxia) }\end{array}$ \\
\hline \multirow{2}{*}{$\begin{array}{l}\text { Riso } \\
(\text { Oryza sativa })\end{array}$} & Diversi geni $B p h$ & $\begin{array}{l}\text { Cicalina del riso } \\
\text { (Nilaparvata lugens) }\end{array}$ \\
\hline & Diversi geni $G m$ & Ditteri del riso \\
\hline \multirow{2}{*}{$\begin{array}{l}\text { Pomodoro } \\
\text { (Solanum lycopersicum) }\end{array}$} & \multirow{2}{*}{ Geni $M i(1$ e 2$)$} & $\begin{array}{l}\text { Afide della patata } \\
\text { (Macrosiphum euphorbiae) }\end{array}$ \\
\hline & & $\begin{array}{l}\text { Mosca bianca del tabacco } \\
\text { (Bemisia tabaci) }\end{array}$ \\
\hline $\begin{array}{l}\text { Melone } \\
\text { (Cucumis melo) }\end{array}$ & $\begin{array}{l}\text { Gene Vat } \\
\text { (resistenza alle punture) }\end{array}$ & $\begin{array}{l}\text { Afide del cotone } \\
\text { (Aphis gossypii) }\end{array}$ \\
\hline $\begin{array}{l}\text { Erba medica } \\
(\text { Medicago truncatula })\end{array}$ & Gene $A I N$ & $\begin{array}{l}\text { Afide "Bluegreen" } \\
\text { (Acyrthosiphon kondoi) }\end{array}$ \\
\hline $\begin{array}{l}\text { Soia } \\
\text { (Glycine max) }\end{array}$ & Diversi geni Rag & $\begin{array}{l}\text { Afide della soia } \\
\text { (Aphis glycines) }\end{array}$ \\
\hline
\end{tabular}

Tabella 2 - Geni identificati per la resistenza ad insetti. Modificata da Broekgaarden et al., 2011 [9]. 


\section{Riferimenti bibliografici}

Tester, M., Langridge, P. 2010, Breeding technologies to increase crop production in a changing world. Science 327(5967): 818-822.

Cattivelli, L., Valè G. 2017, Next Generation Breeding: le conoscenze genomiche rivoluzionano il miglioramento genetico. In: Galbiati, M., Gentile, A., La Malfa, S., Tonelli C. (a cura di). Biotecnologie sostenibili. Edagricole-New Business Media. Collana: Università \& formazione, pp. 1-13.

Sansavini, S., Dondini, L. 2016, Innovazioni nel miglioramento genetico convenzionale $e$ biotecnologico delle piante da frutto. Italus Hortus 23(2): 45-62.

Purcell, A. H., \& Almeida, R. P. 2005, Insects as vectors of disease agents. Encyclopedia of Plant and Crop Science, 10.

Hatchett, J. H. \& Gallun, R. L. 1970, Genetics of the ability of the Hessian fly, Mayetiola destructor, to survive on wheats having different genes for resistance. Anls. Ent. Soc. Am. 63(5): 1400-1407.

Crespo-Herrera, L. A., Garkava-Gustavsson, L., Åhman, I. 2017, A systematic review of rye (Secale cereale L.) as a source of resistance to pathogens and pests in wheat (Triticum aestivum L.). Hereditas 154(1): 14.

Sabbatini, P., Howell, G. S., Herrera, J. C. 2013, Ibridi di Vitis: storia, status e futuro. Italus Hortus 20, 33-43.

Lu, Z. X., Reighard, G. L., Nyczepir, A. P., Beckman, T. G., Ramming, D. W. 2000, Inheritance of resistance to root-knot nematodes (Meloidogyne sp.) in Prunus rootstocks. HortScience 35(7): 1344-1346.

Broekgaarden, C., Snoeren, T. A., Dicke, M., Vosman, B. 2011, Exploiting natural variation to identify insect-resistance genes. Plant Biotech. J. 9(8): 819-825.

Kaloshian, I. 2004, Gene-for-gene disease resistance: bridging insect pest and pathogen defense. J. Chem. Ecol. 30(12): 2419-2438.

Sandhu, S., Kang, M.S. 2017, Advances in Breeding for Resistance to Insects. In: Arora R., \& Sandhu S. (eds) Breeding Insect Resistant Crops for Sustainable Agriculture. Springer, Singapore, pp. 67-99.

Rao, R., Corrado, G., Pennacchio F. 2014, L'incremento della resistenza agli insetti dannosi. In: Rao R. \& Leone A. (a cura di). Biotecnologie e Genomica delle piante. Idelson-Gnocchi, pp. 213-236.

Sonnino, A. 2016, Programmi di difesa integrata delle colture: l'uso di resistenza genetica agli insetti. Energia, ambiente e innovazione, EAI, Roma, 3: 48-53.

Società Italiana di Genetica Agraria (SIGA), 2017 Prima i geni. Liberiamo il futuro dell'agricoltura. http://www.geneticagraria.it/attachment/SocietaScuolaRicerca/Manifesto_Prima_i_Geni.pdf 